\title{
Optical and electrical Barkhausen noise induced by recording ferroelectric domain holograms
}

\author{
Anthony S. Kewitsch, Akira Saito, and Amnon Yariv \\ Department of Applied Physics, California Institute of Technology, Pasadena, California 91125 \\ Mordechai Segev \\ Department of Electrical Engineering, Center for Photonics and Optoelectronics Materials, \\ and Princeton Material Institute, Princeton University, Princeton, New Jersey 08544 \\ Ratnakar R. Neurgaonkar \\ Rockwell International Science Center, Thousand Oaks, California 91360
}

Received July 25, 1994; revised manuscript received March 23, 1995

\begin{abstract}
Ferroelectric domain gratings with periods of the order of an optical wavelength are induced in strontium barium niobate by photorefractive space-charge fields. We measure the Barkhausen noise in current and diffraction efficiency while optically recording domain gratings and show that the two are strongly correlated in time. Significant random depolarization occurs under high-intensity illumination. We deduce the kinetics of the domain inversion process from the shape of the current transients.
\end{abstract}

\section{INTRODUCTION}

We recently reported ${ }^{1-3}$ the generation of spatially periodic, ferroelectric domain gratings induced by optical standing waves in a photorefractive strontium barium niobate (SBN) single crystal. Ferroelectric domain gratings have also been generated by application of external depoling electric field $\mathrm{s}^{4-8}$ during or after optical exposure. In this study we observe electrical Barkhausen noise (characteristic of domain switching) induced by photorefractive space-charge fields and by optical heating as domain holograms are recorded. We also observe noise in the holographic diffraction efficiency as domains switch, which we denote optical Barkhausen noise. These measurements enable us to isolate the role of photorefractive space-charge fields in the generation of dynamic and remnant ferroelectric domain gratings.

\section{BACKGROUND}

The Barkhausen effect was first observed in early research on ferromagnetic domain reversal. ${ }^{9}$ Subsequently, electrical Barkhausen noise was linked to ferroelectric domain switching in barium titanate..$^{9-11}$ As a domain inverts, the changing electric dipole induces a charge on the surface electrodes. This time-varying charge produces a current transient, which is conventionally called a Barkhausen jump. These jumps have been observed in $\mathrm{LiNbO}_{3}$ under intense illumination and at elevated temperatures and were used as evidence for a domain switching contribution to hologram fixing in this material. ${ }^{12}$ Current transients in $\mathrm{BaTiO}_{3}$ during electrical fixing ${ }^{13,14}$ have also been observed. However, the domain switching arising from the uniform depolarization of the crystal during the electrical fixing pulse and the photoinduced space-charge field were not isolated.

\section{THEORETICAL ANALYSIS}

The early measurements and analyses of Barkhausen noise are generally restricted to thin $c$ plates $(\sim 100-$ $\mu \mathrm{m}$ thickness). The domain switching is induced by oscillating external electric fields. Domains typically nucleate below an electrode and subsequently grow to the other electrode. ${ }^{15}$ The crystal is also assumed to be nonconducting, appropriate for crystals such as $\mathrm{BaTiO}_{3}$ in the dark. Because we investigate photoinduced domains, our measurements are performed under illumination and in the presence of mobile charge. We therefore wish to relate the volume of the inverted domains within the illuminated region of the crystal to the measured Barkhausen current noise. We determine this relationship by drawing on the electrostatic theory of a polarized dielectric. The potential and field within a polarized object is equivalent to that produced by a bound volume charge density $\rho_{b}$ :

$$
\rho_{b}=-\nabla \cdot \mathbf{P}_{\mathrm{s}}
$$

and a bound surface charge $\sigma_{b}$ :

$$
\sigma_{b}=\mathbf{P}_{s} \cdot \hat{n} .
$$

That is, the dielectric polarizability represented by $\epsilon$, the low-frequency dielectric constant, is treated as a collection of bound charge residing in vacuum. In these experiments $\mathbf{P}_{\mathrm{s}}$ is identified as the local spontaneous polarization of the ferroelectric phase averaged over a volume much larger than a unit cell and $\hat{n}$ is the unit normal 
vector to the surface of the crystal. The bound charge cannot be removed from a domain interface (where $\mathbf{P}_{\mathrm{s}}$ changes sign); it is permanently attached to the ions responsible for the spontaneous polarization (in the case of $\mathrm{SBN}$ these ions are $\mathrm{Sr}^{2+}, \mathrm{Ba}^{2+}$, and $\mathrm{Nb}^{5+}$ ). In contrast to this bound charge, a free charge can be optically or thermally excited and redistributed, producing photorefractive space-charge fields, for instance. Note that this free charge actually spends the great majority of its time trapped, because the recombination time $(\sim 10$ to $\sim 100 \mathrm{ps)}$ in oxide ferroelectrics such as SBN is extremely short (in this sense, "free charge" is a misnomer). If sufficient mobile charge is present, the bound charge of Eqs. (1) and (2) is compensated by an equal and opposite free-charge density $\rho_{f}$ and surface charge $\sigma_{f}$. This compensating charge is electrostatically trapped at domain walls and other locations where the divergence of $\mathbf{P}_{s}$ is nonzero.

The central theoretical problem is to relate the measured short-circuit current across the crystal to the free and bound charges moving within the crystal. To simplify this analysis, the electrodes across the ferroelectric crystal are treated as infinite conducting sheets short circuited by an ideal current meter $i(t)$ (Fig. 1). Only the center of the crystal is illuminated, so charge carriers do not flow directly from the illuminated region to the electrodes. The induced current is the displacement current $\epsilon \partial E / \partial t$ alone. We adopt a quasi-electrostatic approach, as the frequencies of interest are below $100 \mathrm{kHz}$. Thus currents induced by the magnetic fields associated with moving charges are ignored.

Both the free and the bound charges will contribute to the displacement current measured across the crystal. For example, the free electronic charge is photoexcited and spatially redistributed under illumination. By using the method of images from electrostatics ${ }^{16}$ we obtain an analytical expression for the charges $Q_{A}$ and $Q_{B}$ induced on the conducting plates by the electric field of a point charge $q_{f}$ embedded in a medium with a low-frequency dielectric constant $\epsilon$ :

$$
Q_{A}=-q_{f} \frac{b}{L}, \quad Q_{B}=-q_{f} \frac{a}{L}
$$

where $a$ is the distance from electrode $\mathrm{A}$ to the point charge, $b$ is the distance from electrode $\mathrm{B}$ to the point charge, and $a+b=L$, the distance between the electrodes. From the above analysis it is apparent that the displacement current generated within the crystal under illumination is composed of a free component, even in the absence of domain reversal:

$$
i(t)=\frac{\partial}{\partial t}\left(Q_{B}-Q_{A}\right)=-q_{f} \frac{2}{L} \frac{\partial a}{\partial t} .
$$

$\partial a / \partial t$ gives the component of the free-charge velocity parallel to the surface normal of the electrodes. In general, this free-charge contribution to the displacement current arises from the transport of the free charge as the result of drift, diffusion, and the photovoltaic effect. Note that the displacement current given by Eq. (4) is independent of the low-frequency dielectric constant $\epsilon$.
The second source of current noise is domain switching. The inversion of ferroelectric domains creates characteristically sharp current transients because of the collective displacement of ferroelectric ions, distinguishing this contribution from moving space-charge effects. As an inverted domain nucleates and subsequently grows, a bound charge is created, generating current noise. To relate the measured current across the crystal to the volume of the inverted domain(s) associated with each current spike, we calculate the charges $Q_{A}$ and $Q_{B}$ induced on the conducting plates as a function of the location and size of the inverted, ellipsoidal domain (formally equivalent to charges $q$ and $-q$ separated by a distance $d$, as in Fig. 1). The charge is distributed along the entire domain wall for which $\mathbf{P}_{s}$ is not parallel to the interface. To simplify the analysis we treat the charge as if it were all localized near the foci of the ellipsoidal domain. The induced charge at the electrodes is then

$$
Q_{A}=-q \frac{d}{L}, \quad Q_{B}=q \frac{d}{L}
$$

where $d$ is the distance between the opposite centers of total charge $q$ and $-q$ along the c axis (note that $d$ is slightly smaller than the physical domain length). The induced charge as given by Eqs. (5) is independent of the location of the domain within the crystal and independent of $\epsilon$. In practice, the charge induced by domains near the edges of the finite electrodes does depend on position. We neglect this effect because the crystal is illuminated only at the center in our experiments. We compute the current across the crystal by differentiating the induced charge on the electrodes with respect to time:

$$
i(t)=\frac{\mathrm{d}}{\mathrm{d} t}\left(Q_{B}-Q_{A}\right)=\frac{2}{L}\left(d \frac{\partial q}{\partial t}+q \frac{\partial d}{\partial t}\right) .
$$

$q$ is composed of both the bound charge $q_{b}$ and the free charge $q_{f}$. The free charge is generated on optical illu-
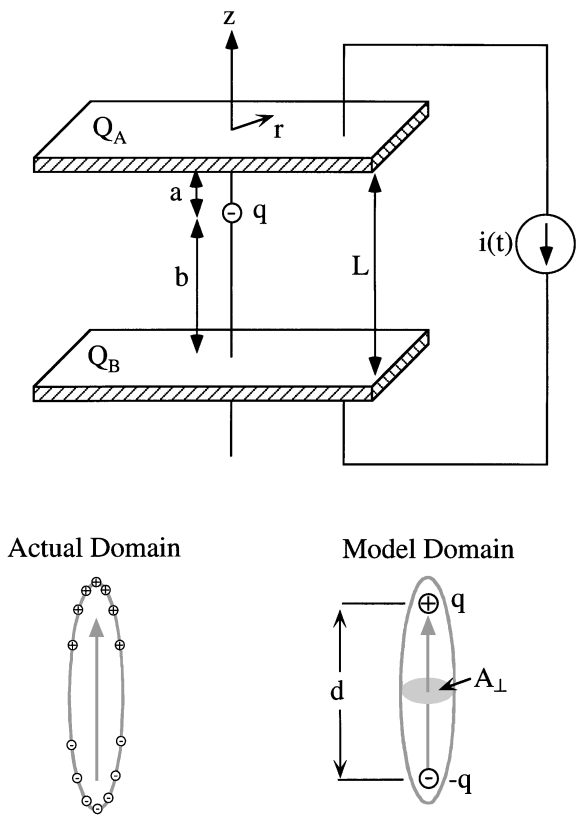

Fig. 1. Theoretical model to relate the measured Barkhausen current to the domain switching dynamics. 
mination and compensates for the bound charge at the domain walls:

$$
q_{b}=\Delta P A_{\perp}=2 P_{d} A_{\perp},
$$

where $A_{\perp}$ is the cross-sectional area indicated in Fig. 1 and $P_{d}$ is the average polarization of the individual domain (typically larger than the macroscopic spontaneous polarization of the entire crystal). On substitution of Eq. (7) into Eq. (6), the transient displacement current that is due to domain switching is

$$
\begin{aligned}
i(t)= & \frac{4}{L} P_{d} A_{\perp} d\left(\frac{\partial \ln A_{\perp}}{\partial t}+\frac{\partial \ln d}{\partial t}+\frac{\partial \ln P_{d}}{\partial t}\right) \\
& +\frac{2}{L}\left(d \frac{\partial q_{f}}{\partial t}+q_{f} \frac{\partial d}{\partial t}\right) .
\end{aligned}
$$

The first term of Eq. (8) represents the sideways growth of domains (perpendicular to the c axis), the second term the nucleation and forward growth (parallel to the c axis), and the third term the change in the individual dipole moment (arising from heating, for example). The last two terms describe the free-charge compensation of the bound charge. They are present in photoferroelectrics because illumination and thermal excitation provide mobile compensating charge.

When a domain grating is written with photorefractive space-charge fields the domains may invert when the field is larger than the local coercive field. However, on inversion, significant depolarization fields equal to $2 P_{d} / \epsilon$ are generated at the head-to-head domain walls. For the resulting domain configuration to be stable, the depolarization fields must also be screened. In fact, the screening of the depolarization fields requires significantly more charge (of the order of $P_{d} / \epsilon E_{c} \sim 10$ times more, where $P_{d} \sim P_{s}$ is the spontaneous polarization and $E_{c}$ is the coercive field) than the initial charge necessary to establish the coercive field. We expect the primary source of this compensating charge to be the photorefractive space charge. However, an additional charge may be provided by thermally or optically excited carriers unrelated to the original spatially periodic photorefractive space-charge field or at preexisting domain walls. This subtle temporal sequence of events in the formation of the space-charge field, lattice distortion, domain switching, and free-charge compensation, can be revealed by examination of the transients in the displacement current.

The depth of spatial modulation of the spontaneous polarization is highly dependent on the optical exposure. This effect results from accelerated free-charge compensation and screening of the ferroelectric dipole interaction ${ }^{17}$ in the presence of illumination. The response time of the compensation is the dielectric relaxation time $\tau_{\text {diel }}$ for diffusion-dominated charge transport (i.e., for grating periods $>$ the Debye screening length, which is of the order of micrometers). Under illumination with light of intensity $I$,

$$
\tau_{\text {diel }}=\frac{\epsilon}{\sigma_{d}+I \sigma_{\mathrm{ph}}}
$$

where $\sigma_{d}$ is the dark conductivity and $\sigma_{\mathrm{ph}}$ is the photoconductivity. For optical intensities of $1 \mathrm{~W} \mathrm{~cm} \mathrm{~cm}^{-2}$ at $45^{\circ} \mathrm{C}$, $\tau_{\text {diel }}$ is $8 \mathrm{~s}$ for Cr-doped SBN:75, whereas in the dark $\tau_{\text {diel }}$ is effectively infinite because of the extremely low dark conductivity. We measure these decay times by monitoring the decay of uniform pyroelectric fields that is due to conduction through the crystal (Fig. 2). The pyroelectric fields are measured with a noncontact electrostatic voltmeter (Trek, Inc.) with an input impedance of $10^{14} \Omega$. Thus, at high optical intensities, we expect that some fraction of Barkhausen noise events may be screened out by opposing free-charge currents.

We estimate the volume of the inverted region by integrating the total charge $Q_{\text {tot }}$ under a current spike. We assume that the current event corresponds to the switching of a single ellipsoidal domain of volume $V$ :

$$
V=\frac{Q_{\mathrm{tot}} L}{6 P_{d}}
$$

Equation (10) also assumes that the compensating free charge exhibits a buildup time [proportional to the dielectric relaxation time of Eq. (9)] that is much slower than the domain switching transient. This is a good assumption for intensities below $100 \mathrm{~W} \mathrm{~cm}{ }^{-2}$, because the high-frequency components of the Barkhausen transient are unscreened by the photoexcited charge for frequencies greater than the inverse dielectric relaxation time. For $10 \mathrm{~W} \mathrm{~cm}{ }^{-2}$ this lower cutoff frequency is estimated from Eq. (9) to be $1 \mathrm{~Hz}$.

\section{EXPERIMENTAL SETUP}

The experimental setup to measure simultaneously the diffraction efficiency of the grating and the current noise is schematically represented in Fig. 3. The experiments are performed on room-temperature poled, Cr-doped SBN:75 and SBN:61 single crystals. The $c$ faces of the crystal are electroded with silver paint. To maintain reproducibility, we occasionally restore the saturated spontaneous polarization by thermalizing the domain structure (heat treating at $350-450{ }^{\circ} \mathrm{C}$ in air) and subsequently electrically poling with $10 \mathrm{kV} \mathrm{cm}^{-1}$ at room temperature. The current noise across the crystal is measured by the input preamplifier of a Stanford Research Systems SR830 lock-in amplifier (1-k $\Omega$ input impedance) or directly on an oscilloscope (1-M $\Omega$ input

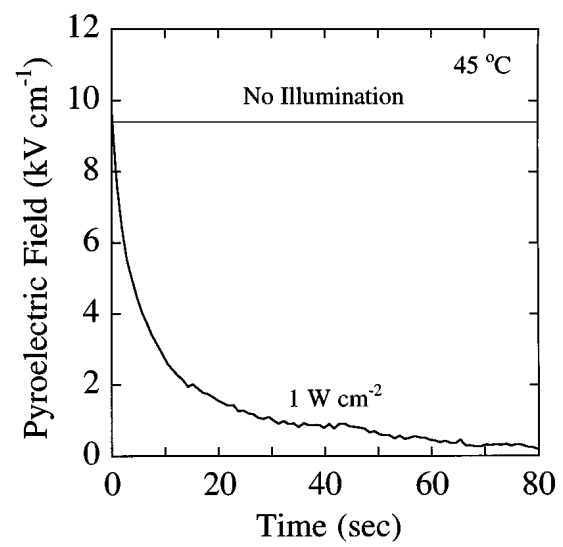

Fig. 2. Decay of pyroelectric fields in the dark and under illumination. The dielectric relaxation time of Cr-doped SBN:75 at $45^{\circ} \mathrm{C}$ is $8 \mathrm{~s}$ at $I_{0}=1 \mathrm{~W} \mathrm{~cm}^{-2}$. 


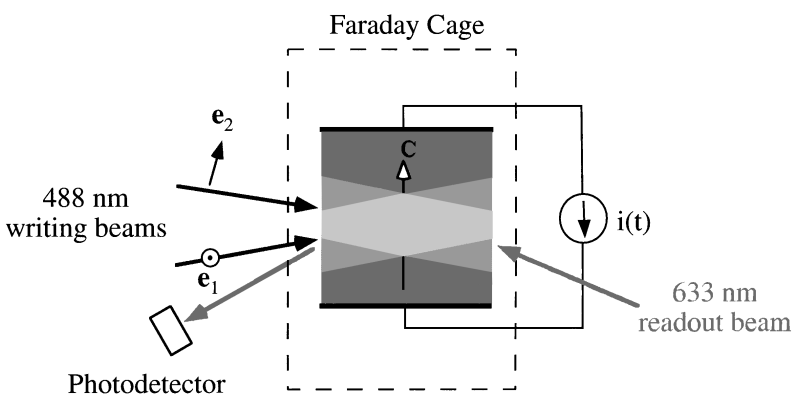

Fig. 3. Experimental setup for writing domain gratings and measuring both the diffraction efficiency and the current across the crystal.

impedance, 500-MHz bandwidth). For high-temporalresolution studies we use a Tektronix 2440 digital storage oscilloscope, and for lower-resolution/long-time studies we use a computer data-acquisition system.

The volume holograms are recorded with two collimated $\mathrm{TEM}_{00}$ single-frequency (488-nm) beams (diameter $1.5 \mathrm{~mm}$ ). The holographic grating (typical period $1.1 \mu \mathrm{m})$ is simultaneously reconstructed with a counterpropagating, $50-\mathrm{mW} \mathrm{cm}^{-2}, 633-\mathrm{nm} \mathrm{He}-\mathrm{Ne}$ laser to monitor the diffraction efficiency. We mechanically adjust the relative polarization of the two recording beams by rotating a half-wave plate in the optical path of one of the beams. This enables us to change the photorefractive space-charge field without changing the total optical intensity, an essential consideration when one is isolating the Barkhausen noise generated by space-charge fields from that generated by optical heating.

\section{EXPERIMENTAL RESULTS}

The first contribution to the displacement current is due to the moving space charge along the holographic grating planes and at the periphery of the optical beams. Inducing a periodic phase shift between the two recording beams at $50 \mathrm{~Hz}$ the spatial phase of the interference pattern is modulated by $\sim 20^{\circ}$. We simultaneously observe $50-\mathrm{Hz}$ noise in the current (Fig. 4). Note that the noise in diffraction efficiency occurs at twice the modulation frequency. We have observed this effect in both SBN:61 and SBN:75. At these low current levels a fraction of the noise current may also result from microdomain alignment in glassy ferroelectrics such as SBN rather than from lattice distortion. Because the microdomains are believed to possess extremely small volumes $\left(\sim 100 \mathrm{~nm}^{3}\right)$, the current induced by inversion of microdomains would also produce an apparently smoothly varying signal. Further research is required to determine the relative magnitudes of the current contributions from microdomain alignment, free-charge transport owing to drift, diffusion, and the photovoltaic effect.

A non-steady-state current has also been reported in the photoconductors $\mathrm{Bi}_{12} \mathrm{SiO}_{20}$ and $\mathrm{Bi}_{12} \mathrm{TiO}_{20}$ on temporal modulation of the space-charge field. ${ }^{18,19}$ In these experiments the displacement current is believed to be negligible compared with the charge transport across the crystal and through the external circuit. The entire crystal is illuminated, so indeed the high photoconductivity of these materials facilitates charge transport from the crystal bulk to the electrodes. In contrast, in our experiments the displacement current is measured because only the center of the crystal is illuminated.

The second and most interesting contribution to the noise current arises directly from the moving bound charge created on domain switching. Figure 5(a) illustrates the noise generated across the crystal during the initial stage of intense optical illumination $\left(\sim 20 \mathrm{~W} \mathrm{~cm} \mathrm{~cm}^{-2}\right)$ with two orthogonally polarized recording beams (one extraordinary, one ordinary, so the electric field vectors of the beams remain orthogonal as they propagate through the uniaxial crystal). At this stage the beams do not

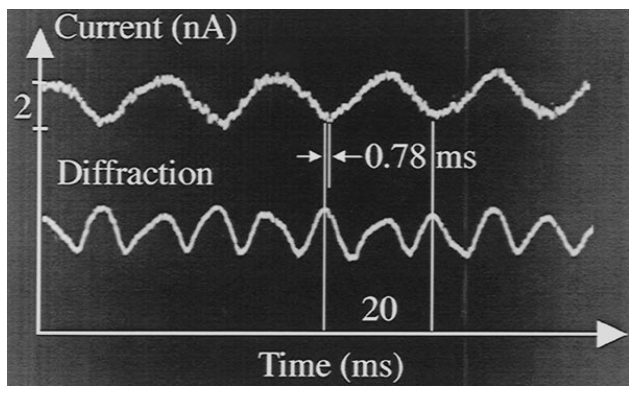

Fig. 4. Noise in current $(50 \mathrm{~Hz})$ induced by an oscillating space-charge field (modulation in diffraction $\sim 0.5 \%$ ). The

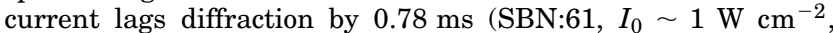
$T=22{ }^{\circ} \mathrm{C}$, thermal steady state)

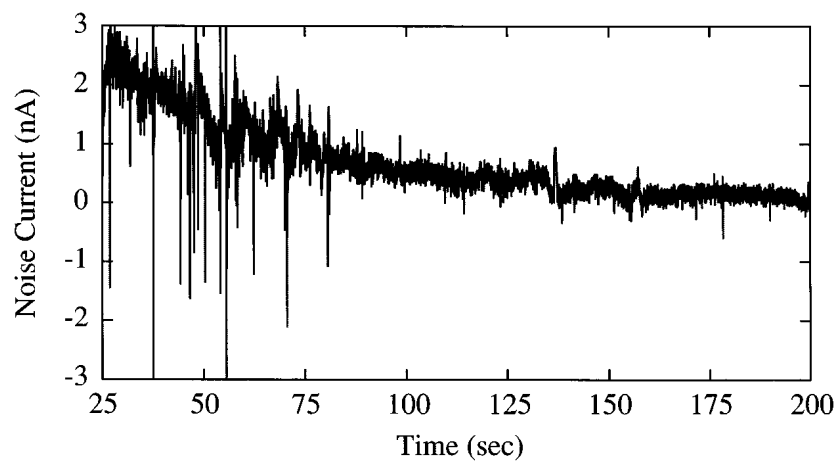

(a)

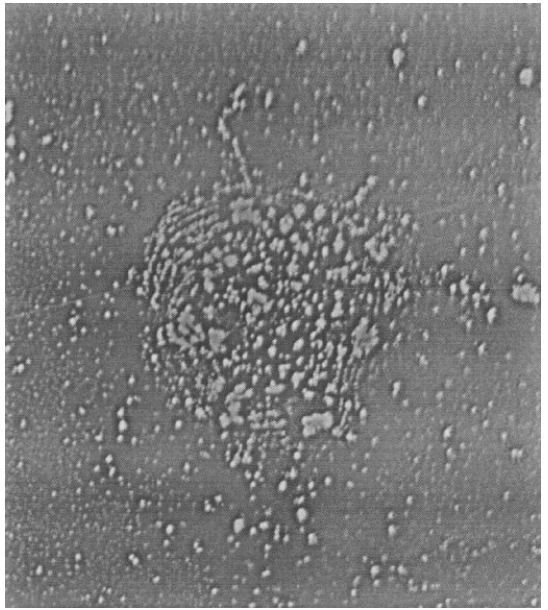

(b)

Fig. 5. (a) Pyroelectric and Barkhausen current caused by optical heating (SBN:75, $I_{0} \sim 20 \mathrm{~W} \mathrm{~cm}^{-2}, T_{\text {ambient }}=22^{\circ} \mathrm{C}$, before thermal steady state), (b) etched $a$ face of a crystal following optical exposure. 
produce a spatially modulated electron density. Illumination begins at $t=0$, and the peak pyroelectric current reaches a value of $250 \mu \mathrm{A}$ on the time scale of milliseconds. The current decays exponentially with a time constant of $400 \mathrm{~ms}$. Figure 5(a) illustrates the subsequent decay, the vertical scale magnified to reveal individual Barkhausen jumps.

If the polarization of each of the two beams instead has a component parallel to the other, producing a photorefractive grating, the noise current does not change significantly. This indicates that most of the current noise during the initial stage of hologram recording with highintensity beams is thermal in origin. Local heating that is due to nonuniform illumination generates local pyroelectric fields. These fields cause domains to invert and produce Barkhausen current spikes [described by the first two terms of Eq. (8)]. Heating also reduces the intrinsic polarization of the individual domains [described by the third term of Eq. (8)] to produce a smoothly varying pyroelectric current. The current jumps are superimposed upon the exponential decay of the pyroelectric current until thermal steady state is reached [Fig. 5(a)].

The rapid increase in current during the first seconds of exposure and the subsequent exponential decay is consistent with the expected pyroelectric current generated along the $c$ axis by optical heating. The heating partially depolarizes the crystal in the region of intense illumination. This contribution to the depolarization is revealed by etching of the entrance $a$ face of SBN crystal [Fig. 5(b)] for $15 \mathrm{~min}$ in a concentrated solution $(37 \%)$ of $\mathrm{HCl}$ at room temperature. We believe that the origin of domain contrast is the selective etching of domain walls, which possess a high concentration of trapped electronic charge that enhances the rate of etching. This is analogous to the contrast mechanism in $\mathrm{BaTiO}_{3}$, in the sense that $+c$ domains (charge compensated by free electrons) etch at a faster rate than $-c$ domains (electrons depleted). ${ }^{20} \mathrm{We}$ subsequently viewed the sample under a transmission optical microscope, using polarized, incoherent light. The circular etched region of Fig. 5(b) corresponds to the 1.5$\mathrm{mm}$-diameter illuminated region. The spatially periodic domain grating was not visible on the etched face at higher magnifications but has been revealed within the bulk by optical means. ${ }^{3}$

Barkhausen jumps arising from the spatially periodic space-charge field alone are also observed. We experimentally isolate the role of the space-charge field in domain switching from thermal effects by maintaining a constant illumination level while tailoring the modulation depth of the interference pattern. First, the crystal is illuminated with two beams of orthogonal polarization and total intensity of $1 \mathrm{~W} \mathrm{~cm} \mathrm{~cm}^{-2}$ for $10 \mathrm{~min}$ to reach thermal steady state. The noise current up to this point is thermal in origin. The polarization of one of the writing beams is then rotated by $90^{\circ}$, establishing the interference pattern. A periodic space-charge grating builds up at $t=0$, as illustrated in the upper trace of Fig. 6. These data reveal the initiation of Barkhausen current events (lower trace) on hologram exposure. The peak magnitude of the space-charge field is $\sim 50 \mathrm{~V} / \mathrm{cm}$, as estimated from the measured diffraction efficiency at $t=2 \mathrm{~s}$ (for $r_{33}=1000 \mathrm{pm} \mathrm{V}^{-1}$, appropriate for a partially depoled crystal). These measurements are repeated with the grating vector perpendicular to the $c$ axis, and the Barkhausen noise transients then vanish. In this geometry the space-charge fields are unable to orient domains periodically.

We deduce that the domain grating rapidly follows the photoinduced space-charge field from the observation of noise on the time scale of the photorefractive buildup time. We call this the dynamic domain grating, in contrast to the more typical remnant domain grating that is used to fix domain holograms permanently. The dynamic domain grating relaxes immediately on the removal of the space-charge field. Ferroelectric hysteresis provides memory so that a fraction of the dynamic domain grating survives as a remnant grating; that is, the electrooptic effect exhibits hysteresis.

We performed identical experiments in a $0.03 \%$ Fe-doped $\mathrm{LiNbO}_{3}$ sample, in the temperature range $25-65{ }^{\circ} \mathrm{C}$. No Barkhausen noise was observed, even in the presence of intense optical illumination. This indicates that the existence of dynamic domain gratings is not a universal property of ferroelectric crystals.

\section{EXPERIMENTAL ANALYSIS}

The microscopic mechanisms of domain reversal can be inferred from the form of the current transients. Barkhausen current jumps were classified into two groups ${ }^{11}$ in the early literature: Jumps of the first type are dome shaped, with a transient characterized by a gentle rise and fall. Jumps of the second type are spikes, with very sharp leading edges (duration of $10 \mu \mathrm{s}$ ) followed by an exponential decay to zero. Jumps of the first type appear for all values of the switching field, whereas jumps of the second type typically appear under strong fields. Jumps of both types typically have smaller average dimensions in fatigued rather than restored crystals. According to Rudyak et al. ${ }^{21}$ the first type of jump is expected during the relatively slow sideways growth of domains. The sharp leading edge of the second type of pulse is associated with a fast nucleation event, and the subsequent exponential decay is associated with the growth of the domain. ${ }^{11}$ The velocity of sideways domain wall motion (parallel to an $a$ axis) is believed to be slower than the forward velocity of domain walls (parallel to a $c$ axis) because of the large depolarization fields established in the former case. The rate of sideways domain growth is expected to increase with optical intensity be-

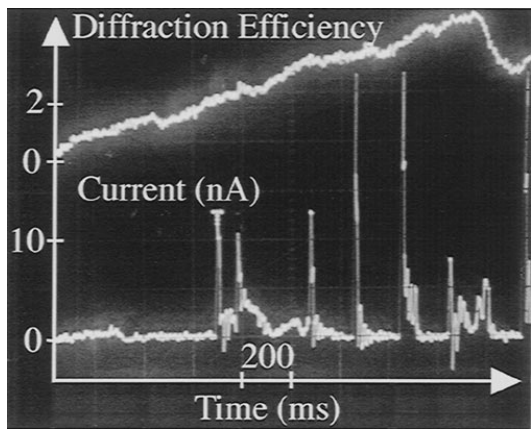

Fig. 6. Noise in current (bottom) and diffraction efficiency (\%, top) as a photorefractive space-charge grating is recorded (SBN:75, $I_{0} \sim 1 \mathrm{~W} \mathrm{~cm}^{-2}, T=30^{\circ} \mathrm{C}$, thermal steady state). 
cause of the availability of mobile charge to screen these depolarization fields.

We observe single-domain switching events of both the first and the second types. We believe that the first type, of 5-ms duration and gradual rise and fall, is associated with the slow sideways growth of a domain [Fig. 7(a)]. The second type has durations of typically $1 \mathrm{~ms}$ and rise times of the order of $10 \mu \mathrm{s}$ [Fig. 7(b)]. The sharp rise corresponds to domain nucleation and the subsequent decay to domain growth. This rise time is in agreement with measurements of single-domain events in $\mathrm{BaTiO}_{3} .{ }^{11}$ In our observations we cannot ignore the possibility of several correlated domain switching events' occurring in a relatively short period of time. In fact, we expect the temporal and spatial correlation of domain switching events. The majority of optically induced current transients in an unfatigued SBN sample display multiple sharp peaks occurring over a period of $100 \mathrm{~ms}$ [Fig. 7(c)].

From Eq. (10) we estimate that the volume of an individual inverted domain associated with a typical current spike in SBN:75 (1-ms duration, 10-nA height, $P_{d} \sim$ $\left.P_{s} \sim 20 \mu \mathrm{C} \mathrm{cm}^{-2}\right)$ is $7 \times 10^{4} \mu \mathrm{m}^{3}$. This volume increases dramatically (by $\sim 2$ orders of magnitude) when multiple domain switching events occur simultaneously. At ambient temperatures current spikes with peak heights as large as $100 \mathrm{nA}$ have been observed in SBN:75, whereas in the SBN:61 sample the spikes are typically 5-10 times smaller in amplitude.

\section{OPTICAL BARKHAUSEN NOISE}

In addition to the strong current noise on high-intensity recording, a previously unreported intensity-dependent noise in diffraction efficiency is also apparent (Fig. 8). Above intensities of $1 \mathrm{~W} \mathrm{~cm}^{-2}$ the noise in diffraction efficiency increases dramatically. We have simultaneously measured both the current and the diffraction efficiency to deduce the origin of this effect (Fig. 8). To study the degree of correlation between these two signals, we compute the cross correlation numerically. The correlation coefficient function between the diffraction $d(t)$ and the Barkhausen current $i(t)$ is defined $\mathrm{as}^{22}$

$$
\rho_{i d}(\tau)=\frac{R_{i d}(\tau)-\mu_{i} \mu_{d}}{\left\{\left[R_{i i}(0)-\mu_{i}^{2}\right]\left[R_{d d}(0)-\mu_{d}^{2}\right]\right\}^{1 / 2}},
$$

where $\mu_{i}$ and $\mu_{d}$ denote the mean values of current and diffraction, respectively, and the cross-correlation function $R_{i d}(\tau)$ is defined as

$$
R_{i d}(\tau)=\lim _{T \rightarrow \infty} \frac{1}{T} \int_{0}^{T} i(t) \mathrm{d}(t-\tau) \mathrm{d} t .
$$

The autocovariance functions for current $R_{i i}(\tau)$ and diffraction $R_{d d}(\tau)$ are defined as

$$
\begin{aligned}
R_{i i}(\tau) & =\lim _{T \rightarrow \infty} \frac{1}{T} \int_{0}^{T} i(t) i(t-\tau) \mathrm{d} t \\
R_{d d}(\tau) & =\lim _{T \rightarrow \infty} \frac{1}{T} \int_{0}^{T} \mathrm{~d}(t) \mathrm{d}(t-\tau) \mathrm{d} t .
\end{aligned}
$$

These correlation integrals can be evaluated in the frequency domain when the data are stationary and ergodic. We low-pass filter the noise current before digital sampling to prevent aliasing. This retains frequency components lower than approximately $50 \mathrm{~Hz}$, which constitute the dominant contribution to the sig-

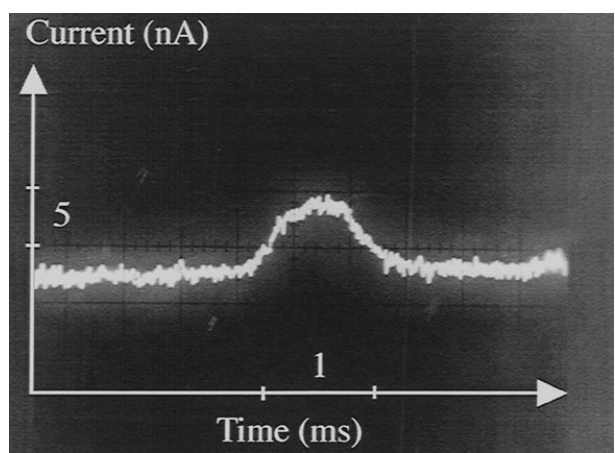

(a)

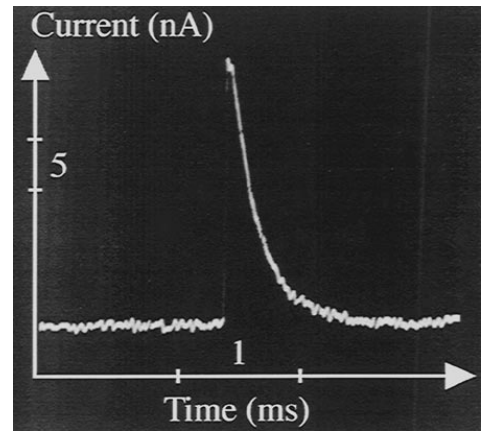

(b)

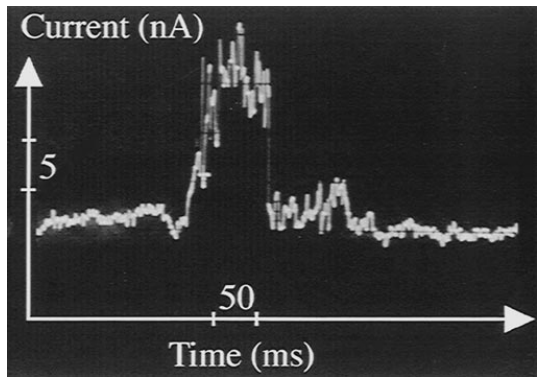

(c)

Fig. 7. Barkhausen current events: single-domain events of (a) type I and (b) type II and (c) multiple-domain switching event (SBN:75, $I_{0} \sim 8 \mathrm{~W} \mathrm{~cm}^{-2}, T_{\text {ambient }}=22^{\circ} \mathrm{C}$, before thermal steady state).

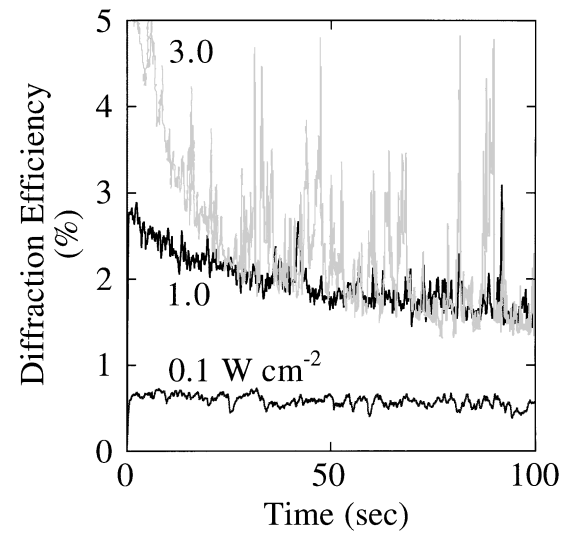

Fig. 8. Intensity dependence of diffraction efficiency noise (SBN:75, $T_{\text {ambient }}=22^{\circ} \mathrm{C}$, before thermal steady state). 


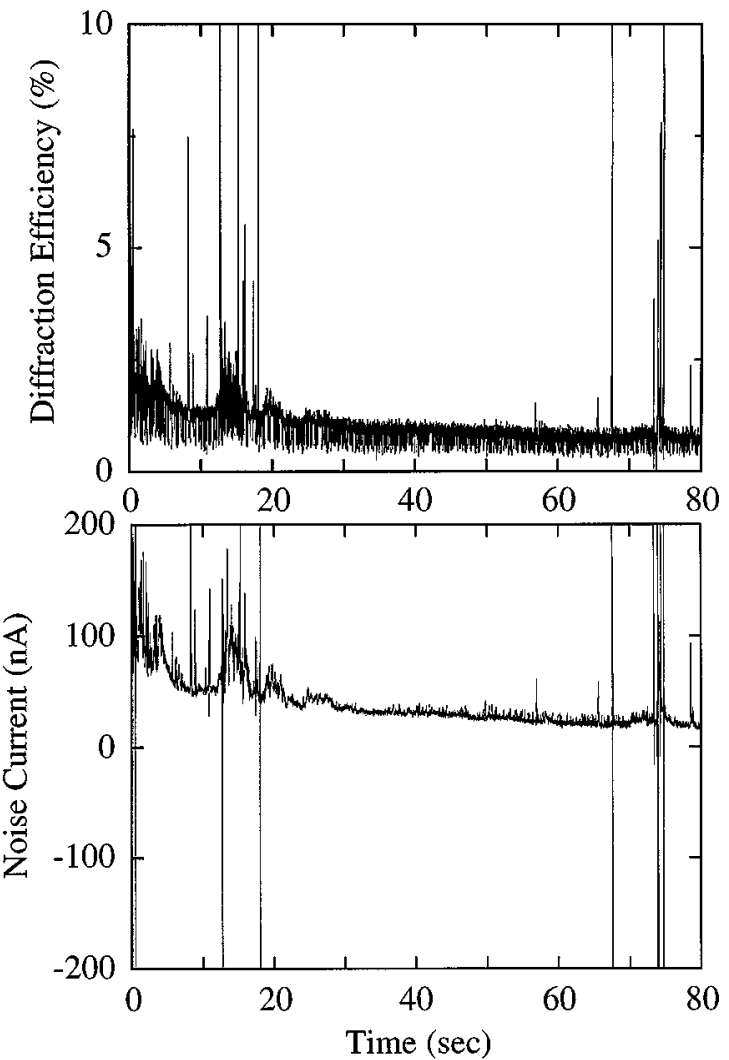

Fig. 9. Long-time temporal correlation of current and diffraction efficiency noise (SBN:75, $I_{0} \sim 20 \mathrm{~W} \mathrm{~cm}^{-2}, T_{\text {ambient }}=22^{\circ} \mathrm{C}$, before thermal steady state).

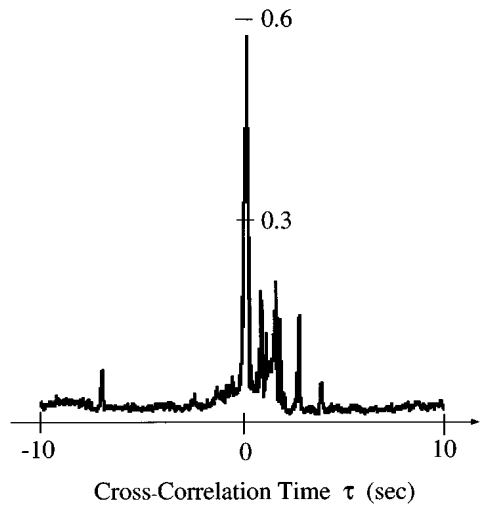

Fig. 10. Correlation coefficient function of current and diffraction efficiency (for data from $t=64$ to $t=80 \mathrm{~s}$ in Fig. 9).

nal in an electrically restored crystal. To compute the correlation, we choose a sample time window containing a large number of events whose average value does not vary appreciably. This sample time window is $16 \mathrm{~s}$, beginning at $t=64 \mathrm{~s}$ in Fig. 9. These data consist of 2048 points sampled every $8 \mathrm{~ms}$. The calculated correlation coefficient function is illustrated in Fig. 10. The large correlation peak at $\tau=0$ indicates that the noise in current and diffraction is correlated for simultaneous events. The series of smaller peaks between $\tau=0$ and $\tau=3 \mathrm{~s}$ indicates a weaker correlation between an initial diffraction peak and later current transients.

The origin of the noise correlation is twofold. The first source of noise correlation arises from domain switching induced by a changing space-charge field, as already dis- cussed in reference to Fig. 6 . In this case, a change in diffraction efficiency triggers a domain switching event. For instance, for the first correlated noise event in Fig. 11 the space-charge field perturbation induces domain switching approximately $200 \mathrm{~ms}$ later. This class of events displays smooth jumps in diffraction efficiency, followed at some later time by sharp jumps in current. The temporal relationship is apparent in Fig. 10 by the delayed sequence of correlation peaks between $\tau=0$ and $\tau=3 \mathrm{~s}$. This figure indicates that, if it were possible to apply a delta function in diffraction efficiency to the crystal, we would subsequently observe a series of noise spikes in the current, whose probability of occurrence decays within a characteristic time of $\sim 3 \mathrm{~s}$. That is, Fig. 10 is the impulse response of the ferroelectric distortion under periodic space-charge fields. We believe that a primary source of this noise is the instability of the spatial phase and amplitude of the optical interference pattern during optical heating. These noise events vanish once thermal steady state is reached. However, if the optical interference pattern is subsequently perturbed, a sequence of current spikes will be generated as the new domain grating is recorded.

Secondly, a large fraction of correlated noise events in current and diffraction occur simultaneously. In this case, the Barkhausen current jump is initiated before the space-charge field has changed, so the noise in diffraction efficiency is triggered by domain switching (e.g., the second event of Fig. 11). Accordingly, this class of events is unrelated to noise in the optical interference pattern. The events are represented by a strong correlation peak ( $\rho_{i d}=0.55$ at $\tau=0$ in Fig. 10) and characterized by abrupt transients in both diffraction efficiency and current. This noise also vanishes after thermal steady state is reached, indicating that the domain switching responsible for the noise in diffraction efficiency is initiated by random depolarization that is due to optical heating, rather than by periodic space-charge fields. This is to be expected because the domain switching arising from optical heating is much more significant than that induced by the space-charge fields. The relative contributions are apparent when one notes the much stronger Barkhausen noise in Fig. 9 than in Fig. 6.

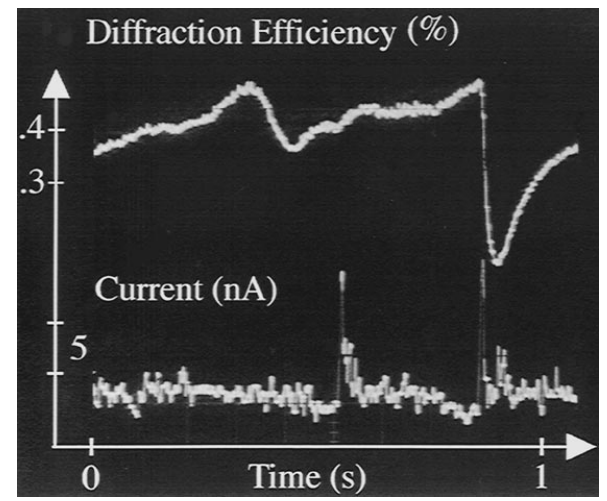

Fig. 11. Two characteristic noise events in current and diffraction efficiency. The first event is characteristic of a fluctuation in the space-charge field initiating domain reversal. The second event is characteristic of noise in diffraction efficiency induced by domain switching (SBN:75, $I_{0} \sim 8 \mathrm{~W} \mathrm{~cm}^{-2}, T_{\text {ambient }}=22^{\circ} \mathrm{C}$, before thermal steady state). 
We propose that this second source of noise correlation is primarily the result of a noise current perturbing the free charge transport during dynamic hologram formation. This is analogous to the noise in dynamic beam coupling that would be induced by a fluctuating external field applied across the illuminated region of the crystal. Indeed, the photorefractive band-transport model predicts that moderate electrical noise fields across the grating volume will introduce dramatic noise in the dynamic beam coupling. Once thermal steady state is reached, this noise current driven by nonuniform optical heating vanishes. In addition, the domain volume passes through the paraelectric phase for a short period of time on switching between the two ferroelectric orientations, which results in a large transient index change $(\sim 0.02$ at $25^{\circ} \mathrm{C}$ ). The entire domain volume becomes an efficient scatterer of light for a fraction of the switching time. In a separate experiment we monitored the beam fanning during these high-intensity exposures and observed an identical correlation in the total intensity of the fanning beam and the noise current.

\section{CONCLUSIONS}

In conclusion, we have observed Barkhausen current noise associated with ferroelectric domain reversal induced by photorefractive space-charge fields. We find a strong correlation between the current and the diffraction efficiency noise under high-intensity optical exposures. Furthermore, the rapid buildup of current noise on hologram recording indicates that a dynamic domain grating is formed. This technique enables us to isolate the primary factors responsible for optically induced domain reversal, namely, high intensities and space-charge fields.

\section{ACKNOWLEDGMENTS}

The authors express gratitude to the anonymous reviewers for their insightful comments. This research is supported by the Advanced Research Projects Agency, the U.S. Air Force Office of Scientific Research, and the U.S. Office of Naval Research.

\section{REFERENCES}

1. A. S. Kewitsch, M. Segev, A. Yariv, and R. R. Neurgaonkar, "Selective page-addressable fixing of volume holograms in $\mathrm{Sr}_{0.75} \mathrm{Ba}_{0.25} \mathrm{Nb}_{2} \mathrm{O}_{6}$," Opt. Lett. 18, 1262-1264 (1993).

2. A. S. Kewitsch, M. Segev, A. Yariv, G. J. Salamo, T. W. Towe, E. J. Sharp, and R. R. Neurgaonkar, "Tunable quasiphase matching using dynamic domain gratings induced by photorefractive space charge fields," Appl. Phys. Lett. 64, 1023-1025 (1994).
3. A. S. Kewitsch, M. Segev, A. Yariv, G. J. Salamo, T. W. Towe, E. J. Sharp, and R. R. Neurgaonkar, "Ferroelectric domain gratings in strontium barium niobate induced by photorefractive space charge fields," Phys. Rev. Lett. 73, 1174-1177 (1994).

4. F. Micheron and G. Bismuth, "Electrical control of fixation and erasure of holographic patterns in ferroelectric materials," Appl. Phys. Lett. 20, 79-81 (1972).

5. Y. Qiao, S. Orlov, D. Psaltis, and R. R. Neurgaonkar, "Electrical fixing of photorefractive holograms in $\mathrm{Sr}_{0.75} \mathrm{Ba}_{0.25} \mathrm{Nb}_{2} \mathrm{O}_{6}$," Opt. Lett. 18, 1004-1006 (1993).

6. M. Horowitz, A. Bekker, and B. Fischer, "Broadband secondharmonic generation in $\mathrm{Sr}_{x} \mathrm{Ba}_{1-x} \mathrm{Nb}_{2} \mathrm{O}_{6}$ by spread spectrum phase matching with controllable domain gratings," Appl. Phys. Lett. 62, 2619-2621 (1993).

7. M. Horowitz, A. Bekker, and B. Fischer, "Image and hologram fixing method with $\mathrm{Sr}_{x} \mathrm{Ba}_{1-x} \mathrm{Nb}_{2} \mathrm{O}_{6}$ crystals," Opt. Lett. 18, 1964-1966 (1993).

8. F. Kahmann, R. Pankrath, and R. A. Rupp, "Photoassisted generation of ferroelectric domain gratings in SBN," Opt. Commun. 107, 6-10 (1994).

9. V. M. Rudyak, "The Barkhausen effect," Sov. Phys. Usp. 13, 461-479 (1971).

10. R. R. Newton, A. J. Ahearn, and K. G. McKay, "Observation of the ferro-electric Barkhausen effect in barium titanate," Phys. Rev. 75, 103-106 (1949).

11. A. G. Chynoweth, "Barkhausen pulses in barium titanate," Phys. Rev. 110, 1316-1332 (1958).

12. V. I. Kovalevich, L. A. Shuvalov, and T. R. Volk, "Spontaneous polarization reversal and photorefractive effect in single-domain iron-doped lithium niobate crystals," Phys. Status Solidi (a) 45, 249-252 (1978).

13. R. S. Cudney, J. Fousek, M. Zgonik, P. Günter, M. H. Garrett, and D. Rytz, "Photorefractive and domain gratings in barium titanate," Appl. Phys. Lett. 63, 3399-3401 (1993).

14. R. S. Cudney, J. Fousek, M. Zgonik, P. Günter, M. H. Garrett, and D. Rytz, "Enhancement of the amplitude and lifetime of photoinduced space-charge fields in multidomain ferroelectric crystals," Phys. Rev. Lett. 72, 3883-3886 (1994).

15. R. C. Miller, "Some experiments on the motion of $180^{\circ}$ domain walls in $\mathrm{BaTiO}_{3}$," Phys. Rev. 111,736-739 (1958).

16. J. D. Jackson, Classical Electrodynamics (Wiley, New York, 1975).

17. V. M. Fridkin, Photoferroelectrics (Springer-Verlag, Berlin, 1979).

18. M. P. Petrov, I. A. Sokolov, S. I. Stepanov, and G. S. Trofimov, "Non-steady-state photo-electromotive-force induced by dynamic gratings in partially compensated photoconductors," J. Appl. Phys. 68, 2216-2225 (1990).

19. I. A. Sokolov and S. I. Stepanov, "Non-steady-state photoelectromotive force in crystals with long photocarrier lifetimes," J. Opt. Soc. Am. B 10, 1483-1488 (1993).

20. J. A. Hooton and W. J. Merz, "Etch patterns and ferroelectric domains in $\mathrm{BaTiO}_{3}$ single crystals," Phys. Rev. 98, 409-413 (1955).

21. V. M. Rudyak, A. Y. Kudzin, and T. V. Panchenko, "Barkhausen jumps and stabilization of the spontaneous polarization of single crystals of $\mathrm{BaTiO}_{3}$," Sov. Phys. Solid State 14, 2112-2113 (1973).

22. J. S. Bendat and A. G. Piersol, Engineering Applications of Correlation and Spectral Analysis (Wiley-Interscience, New York, 1993). 